\title{
Transdifferentiation Meets Next-generation Biotechnologies
}

\author{
Xiaoshan $\mathrm{Ke}^{\mathrm{a}, \mathrm{b}}$, Abhimanyu Thakur ${ }^{\mathrm{a}, \mathrm{b}}$ and Huanhuan Joyce Chen ${ }^{\mathrm{a}, \mathrm{b}, *}$ \\ ${ }^{a}$ The Pritzker School of Molecular Engineering, The University of Chicago, IL, USA \\ ${ }^{\mathrm{b}}$ The Ben May Department for Cancer Research, The University of Chicago, IL, USA
}

Pre-press 6 January 2022

Published 13 January 2022

\begin{abstract}
Transdifferentiation is the process of converting terminally differentiated cells to another cell type. Being less time-consuming and free from tumorigenesis, it is a promising alternative to directed differentiation, which provides cell sources for tissue regeneration therapy and disease modeling. In the past decades, transdifferentiation was found to happen within or across the cell lineages, being induced by overexpression of key transcription factors, chemical cocktail treatments, etc. Implementing next-generation biotechnologies, such as genome editing tools and scRNA-seq, improves current protocols and has the potential to facilitate discovery in new pathways of transdifferentiation, which will accelerate its application in clinical use.
\end{abstract}

Keywords: Transdifferentiation, directed differentiation, stem cells, tissue regeneration, gene editing, whole-genome sequencing

\section{INTRODUCTION}

The human body is composed of trillions of cells with various shapes, sizes, and functions. During the development, cell changes from one type to a more specialized type based on the body's needs. A comprehensive understanding of the mechanisms behind cell fate decisions enables induction of specific cell types and helps researchers to optimize model diseases and develop tissue regeneration therapies. Directed differentiation, defined as the process of inducing a particular type of cells from pluripotent cells, is one of the methods developed for cellular differentiation studies; however, there remains disadvantages. Whereas transdifferentiation, defined as the process of directly converting terminally differentiated cells into another type of cells, bypasses the pluripotent stage and brings several benefits such as less time consumption and lower chances of tumorigenesis. Here we summarized the current progress

\footnotetext{
*Correspondence to: Huanhuan Joyce Chen. E-mail: joycechen @uchicago.edu.
}

researchers have made in the field, including transdifferentiation within or across the cell lineages. The six significant lineages discussed here are mesenchymal lineage, hematopoietic lineage, cardiac lineage, hepatic lineage, pancreatic lineage, and neural lineage, which covered cells originated from three different germ layers (Fig. 1). Furthermore, we discussed the applications of next-generation technologies in the study of transdifferentiation. Next-generation genome editing technologies and sequence analysis have emerged as promising tools for improving the current transdifferentiation protocols by either enhancing conversion efficiency or revealing novel pathways. In the future, implementing these new technologies would help accelerate the development of transdifferentiation for clinical use.

\section{DIRECTED DIFFERENTIATION VS. TRANSDIFFERENTIATION}

Stem cells gained significant attention in the field of tissue engineering and regenerative medicine in the past years. Featuring the abilities of self-renewal 


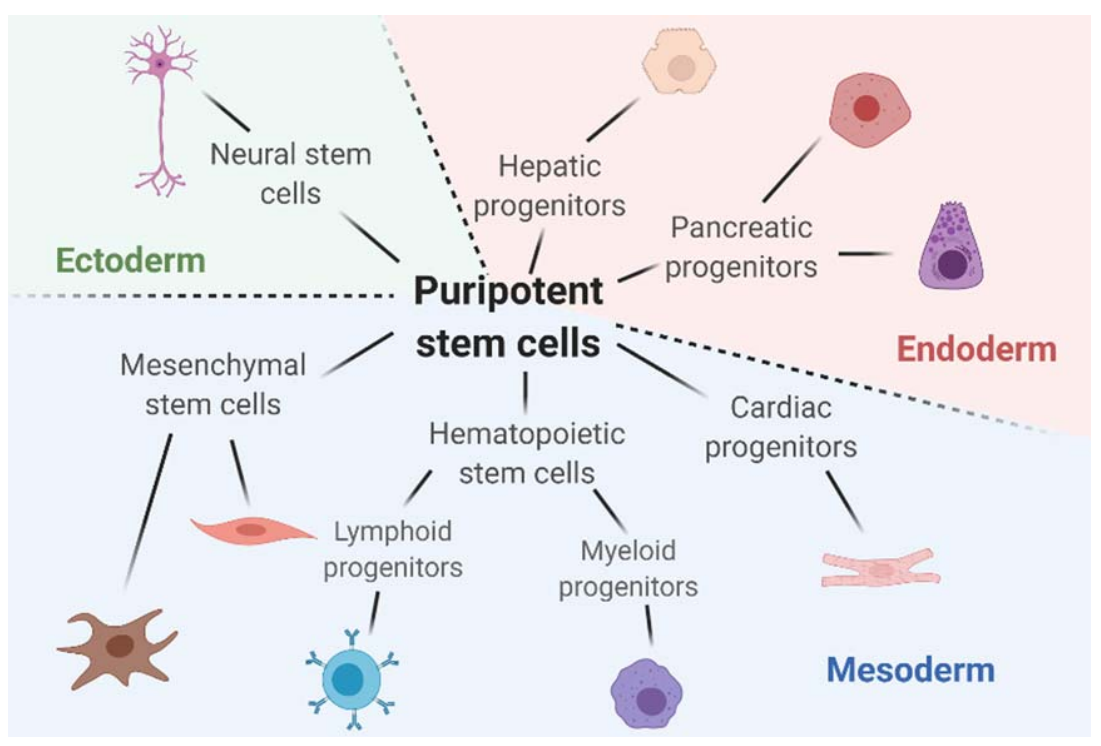

Fig. 1. A summary on the six major lineages discussed in this review and the representative cells in each lineage. Figure is created with BioRender.com.

and differentiation into almost all types of specialized cells, embryonic stem cells (ESC) has been one of the major cell types for the stem cell studies. However, the ethical issues have been one of the main barriers when using ESCs that come from the human embryos. To circumvent this problem, researchers developed methods to convert differentiated cells back to embryonic stem cell-like cells, which are called induced pluripotent stem cell (iPSC) [1]. The groundbreaking work of inducing pluripotent stem cells from mouse fibroblast cells was done by Takahashi and Yamanaka in 2006 [2]. They found that transcription factors Oct4, Sox2, Klf4, and c-Myc played important roles in inducing the pluripotency of the somatic cells. These four transcription factors later became the basic combination of regulators in iPSC studies. By fine-tuning the expression level of each transcription factors, adding or excluding one or more of them, the protocols were improved to get higher efficiency for reprogramming different types of differentiated cells [3-5]. Human fibroblast cells were successfully reprogrammed to iPSCs in 2007 by Yamanaka group and Thomson group, using retrovirus and lentivirus vectors as transduction platforms, respectively [6, 7]. More methods were developed to avoid problems caused by potential transgene reactivation, such as cooperating with a temperature-sensitive mutant of the Sendai virus to control the expression of regulators [8]. Later, cells from tissues other than skin fibroblasts, such as urine tissues, peripheral blood, and hair, were also used as sources to generate iPSCs [8-10]. However, no matter the combinations of regulators, delivery methods, or cell sources used in the reprogramming process, applications of both ESCs and iPSCs in either cellular therapy or tissue engineering involve directed differentiation from their pluripotent stage, which requires various amount of time for generating different cell types and can be extremely time-consuming. The current protocols for directed differentiation in some cell lineages still need improvements to achieve higher efficiency and better cell maturity. Moreover, researchers reported that human tumor cells and iPSC-differentiated cells express similar significant genes [11], indicating that pluripotency brings tumorigenicity. Although several improvements were made to track and prevent tumorigenesis in the directed differentiated cell-derived tissues [12, 13], tumorigenesis has become a primary concern when translating iPSC-derived cells for cellular therapies or clinical applications.

Interestingly, bypassing the pluripotency stage can be one of the solutions to the above issue. Besides directed differentiation, transdifferentiation, also termed direct cell reprogramming, becomes a promising alternative in the field. Unlike iPSCs, cells are directly converted from one fully differentiated cell type to another, either within or across the germ layers. The earliest findings of in vitro direct reprogramming of differentiated cells can be traced back to 1987, when Davis, Weintraub, and Lassar converted mouse embryonic fibroblasts to myoblasts by trans- 
fecting the cell line with MyoD expression vehicles [14]. Enforcing transcription factors level by introducing an exogenous transgene into the cells is a standard tool to initiate the transdifferentiation process [15]. The critical transcription factors usually differ when using different cell sources or targeting different cell types [16]; therefore, understanding cell fate decisions' mechanisms is crucial for inducing transdifferentiation experimentally.

Transdifferentiation saves time and money compared to directed differentiation by integrating two steps into one. However, during the transition across a longer epigenetic distance, the efficiency of transdifferentiation gets lower [17]. Besides using transcription factors with better regulatory ability, manipulating endogenous genes with gene-editing tools is another good way to facilitate the transdifferentiation process [18]. Researchers have improved the efficiency of transdifferentiating human fibroblasts to dopaminergic neurons by promoting G1 arrest via different methods, suggesting that cell cycle arrest and appropriate extracellular environment play significant roles in the conversion process [19]. A relatively lower efficiency had been one of the reasons that impeded clinical applications of transdifferentiation. There were 11 approved clinical trials to date involving human PSC-based therapies registered at the National Institutes of Health website [20], whereas no transdifferentiated cell-based therapies have yet to be approved [15]. As the fast development of direct reprogramming technologies in recent years, the shortcomings of transdifferentiation are gradually overcame, and their regenerative medicine applications are promising.

\section{TRANSDIFFERENTIATION WITHIN OR ACROSS LINEAGES}

In the well-known Waddington's epigenetic landscape, the process of a cell differentiated from a pluripotent state to a committed state is depicted like a ball rolling down a hill until it reaches its minimum energy at the bottom (Fig. 2). For transdifferentiation to happen, the cell must overcome the kinetic barrier it erected to maintain the local energy minimum, including the key proteins, cell cycle, and extracellular environment [21]. The difficulty to overcome this barrier varies by the distance between source cells and targeted cells. Conversions are called "small jumps" when both the source cells and the targeted cells are originated from the same germ layer. On the other

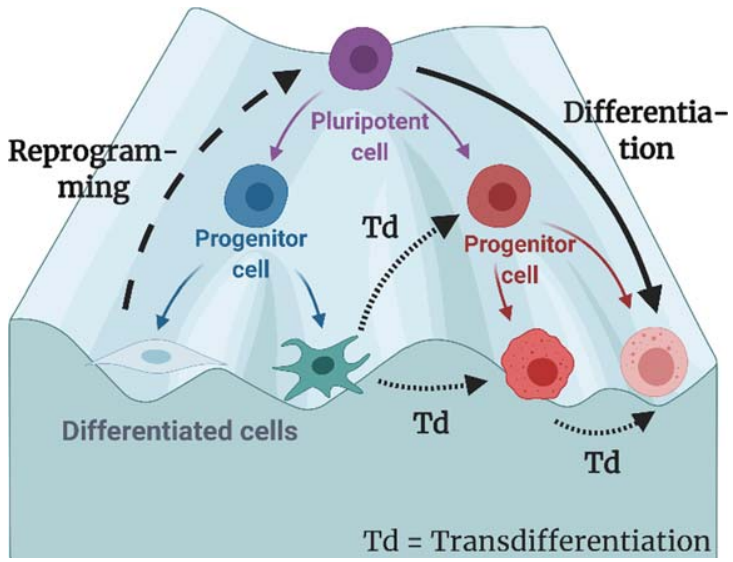

Fig. 2. The process of differentiation, reprogramming, and transdifferentiation (direct reprogramming) depicted on the classical Waddington Landscape. In this review, transdifferentiation are defined as the process in which the origin cells are fully differentiated, and the target cells do not have pluripotency. Figure is created with BioRender.com.

hand, "large jumps" refer to those that transdifferentiate across different germ layer.

The conversion from fibroblasts to neurons is one example of the "large jumps". Neurogenerative diseases are among the most severe health problems worldwide and have gained researchers great attention for developing modeling systems and effective therapies. Wernig et al. first discovered that the combination of transcription factors Brn2, Ascl1, and Mylt1 (BAM) are sufficient to convert mouse embryonic fibroblast to neurons in 2010 [22]. Conversion from human fibroblasts was achieved in 2011 by addition of either microRNA or other transcription factors to BAM [23, 24]. Without the introduction of ectopic genes, microRNAs alone [25] or chemical cocktails alone [26] are capable of inducing these "large jumps". Several specific neuronal subtypes were derived for application in regenerative medicine targeting different disease, such as dopaminergic neurons [27], motor neurons [28], and GABAergic interneurons [29]. Besides fibroblasts, "small jumps" from astrocytes to neurons in vitro and in vivo were also studied and reported by various groups [30]. In 2018, Tanabe et al. converted purified $\mathrm{T}$ cells into functional neurons via episomal vectors. Compared to fibroblast and astrocytes, these human peripheral blood cells are better donor cells without invasive biopsies and extensive cell expansion in vitro [31]. Induced unipotent neural cells were terminally differentiated which motivated researchers to explore transdifferentiation from somatic cells to 


\section{Important studies on transdifferentiation in the past decades}

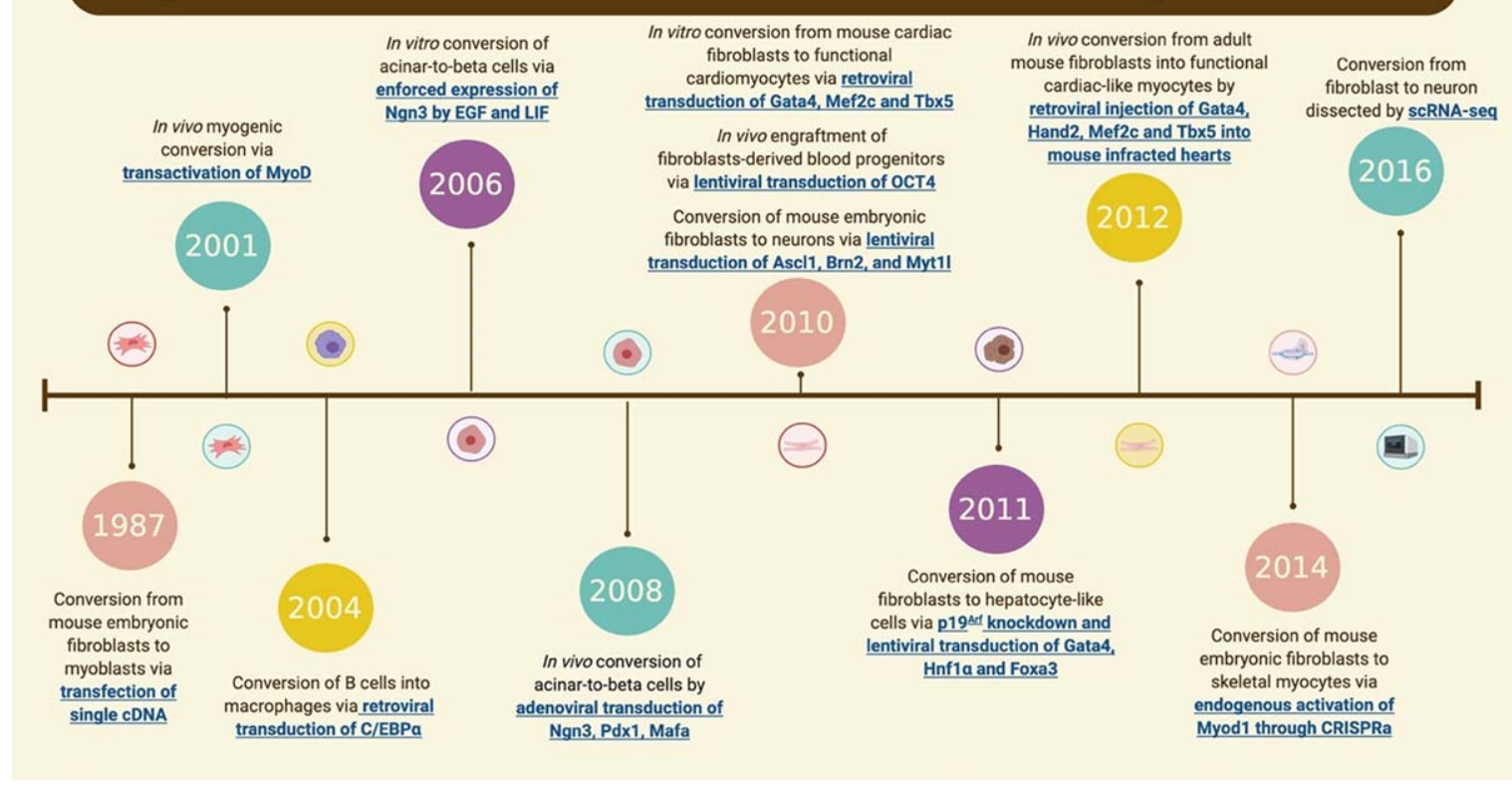

Fig. 3. Timeline of the important works in the field of transdifferentiation the past decades. Figure is created with BioRender.com.

multipotent neural stem cells [32-35]. In more recent studies, researchers came up with strategies using small molecules to interact with endogenous factors and induce intrinsic neural programs [36] or using plasmid-based transfection to reduce the risk of random genomic integration [37].

Last but not least, transdifferentiation involving cells derived from endoderm such as hepatocytes and pancreatic cells has also been studied and characterized in vitro and in vivo. In 2011, two groups induced hepatocyte-like cells from mouse fibroblasts by defined factors. Huang et al. transduced the tail-tip fibroblasts with GATA4, Hnf $1 \alpha$, and Foxa3 and inactivated p19Arf [38], whereas Sekiya and Suzuki used combinations of $\mathrm{Hnf} 4 \alpha$ plus either Foxa1, Foxa2, or Foxa3 to achieve the derivation of the hepatocytelike cells [39]. Later, stable and expandable induced hepatic stem cells were obtained from mouse embryonic fibroblasts by overexpression of $\mathrm{Hnf} 1 \beta$ and Foxa3 [40]. In 2014, conversion from human fibroblasts was achieved via the lentiviral expression of Foxa3, Hnfl $\alpha$, and Hnf4 $\alpha$ [41]. Strategies such as delivering transcription factor via episomal plasmidbased method [42] and using synthetically modified mRNAs to overexpress the factors [43] helped reduce the risk of mutagenesis and genotoxicity. Nakamori et al. improved conversion efficiency from human fibroblasts to hepatocyte-like cells using the combination of Atf5, Prox1, Fxoa2, Foxa3, and Hnf4 $\alpha$ [44]. Interestingly, Song et al. successfully converted profibrogenic myofibroblasts into hepatocyte-like cells [45], which provided a new idea to treat liver fibrosisrelated disease. Meanwhile, pancreatic cells were subjected to different mechanisms to induce the conversion. Baeyens and colleagues started their study in acinar-to-beta cell conversion via activation of STAT3 signaling in 2006 [46]. In 2008, Zhou et al. used the combination of Ngn3, Pdx1, and Mafa to convert acinar cells to beta-cells in vivo [47]. Later, $\mathrm{Li}$ et al. reported that acinar cells could also be converted to somatostatin gamma-like cells by Ngn3 and glucagon alpha-like cells by Ngn3 and Mafa in 2014 [48]. Both Dhawan et al. and Papizan et al. successfully converted beta cells to alpha cells in vivo in 2011 by repressing the Arx promoter through DNA methylation [46].

\section{CURRENT PROGRESS OF TRANSDIFFERENTIATION}

Davis, Weintraub, and Lassar did an acknowledged milestone of transdifferentiation in the lab in 1987 by converting fibroblasts to myoblasts (Fig. 3) $[16,49]$. Based on a previous experiment of deriv- 
ing myogenic, adipogenic, and chondrogenic clones from embryonic mouse fibroblast line [50], they isolated the cDNA clones representing the sequences absent in fibroblast cells but present in myogenic cell lines. While transfecting vectors carried several different cDNA candidates into the fibroblast cells, they figured the most critical sequence for converting fibroblast to myoblast and named the gene that encodes this sequence MyoD1 [14]. MyoD1 was proved to be the master gene regulator for myogenesis [51] and was later used to activate muscle-specific genes in various cell lines [52-55]. In vivo myogenic conversion was also achieved by Del Bo et al. when they forced MyoD expression of the NIH3T3 fibroblasts by transactivating tetracycline and implanted these fibroblasts into the mice [56]. Bichsel et al. improved the MyoD protein delivery process by applying a P.aeruginosa bacteria protein injection system, directly injecting proteins into the cell cytoplasm. This method sufficiently induced myogenic conversion of fibroblasts and was able to generate multinucleate myotubes [57]. To further enhance the degree of transdifferentiation, Boularaoui et al. manipulated several signaling pathways in addition to forced MyoD1 expression in human dermal fibroblasts. Fibroblasts were exposed to various protein ligands or small molecules and achieved the highest conversion rate of $97.7 \%$ when implementing TGFß/activin inhibition [58]. In more recent studies, fibroblasts were converted to expandable muscle progenitor cells by certain combinations of transcription factors, revealing an essential role of Pax3 in reprogramming somatic cells into muscle progenitor cells $[59,60]$. For example, in a 2019 study, Hwang et al. combined Pax3 with Sox2, Klf4, c-Myc and Esrrb to convert mouse embryonic fibroblasts into myogenic lineage cells that can stably proliferate and further differentiate into muscle fibers [61].

Dysfunction of cardiomyocytes is the primary cause of heart failure, leading to massive deaths worldwide every year. Due to the limited capacity of cardiomyocytes regenerating themselves, researchers turned to investigate alternative strategies for cardiac regeneration [62], such as the derivation of cardiac myocytes via transdifferentiation. Before 2010, partial direct conversion of fibroblasts into cardiac myocyte-like cells was achieved by either treatment with TGF- $\beta$, electrostimulation, exposure to 5-aza, or lentiviral delivery of myocardin [63]. In 2010, Ieda et al. used the combination of GATA4, MEF2C, and TBX5(GMT) to convert mice cardiac fibroblasts into functional cardiomyocytes [64]. The induced cardiomyocytes had a similar gene expression profile to neonatal cardiomyocytes and were able to contract spontaneously, and the whole conversion process was relatively rapid. By adding Hand2 to the GMT combination (GHMT), more than one group achieved a higher efficiency of cardiac reprogramming [65]. In 2013, Hirai et al. fused the MyoD transactivation domain to GHMT to enhance the generation of beating clusters of induced cardiomyocytes [66]. Incidentally, direct conversion from human fibroblast to cardiac myocytes was reported by three different groups, using extra transcription factors in addition to GMT or GHMT. Besides transcription factors, muscle-specific microRNAs and pure chemical cocktails were also proven to induce the generation of functional cardiomyocytes [67, 68]. Recently, cardiac reprogramming has been expanded to conversion from fibroblasts to endothelial cells, from endothelial cells to cardiomyocytes, and from cardiomyocytes to pacemaker myocytes [63]. However, the completeness and efficiency of these transdifferentiation processes still need to be improved [15].

The transdifferentiation technology has been developed and applied to convert cell types in the blood lineage. As early as 1982, Boyd and Schrader modified pre-B lymphoma cell lines with the demethylating drug 5-azacytidine and successfully derived macrophage-like cell lines from it [69]. After that, more and more studies revealed the possibility of lineage switches within the hematopoietic system [70]. Thomas Graf and his group are among the pioneers in reprogramming blood cells. In 2004, they directly reprogrammed differentiated B cells into macrophages by enforcing the expression of $\mathrm{C} / \mathrm{EBP} \alpha$ and $\mathrm{C} / \mathrm{EBP} \beta$ [71]. C/EBPs inhibited the transcription factor Pax5, whose absence was found to be essential in various B lineage cell commitments and tissue reconstitution by previous studies $[72,73]$. Another vital transcription factor is PU.1, which promotes macrophages differentiation when expressed at a high level [74]. In 2006, the group developed a method to reprogram committed $\mathrm{T}$ cell progenitors to macrophages and dendritic cells by the combination of $\mathrm{C} / \mathrm{EBP} \alpha$ and PU.1 transcription factors [75]. Two years later, they successfully converted mesenchymal stem cells derived fibroblasts into macrophages by retroviral expression of C/EBPs and PU.1 [76]. In 2010, Szabo et al. converted human neonatal and dermal fibroblasts into multilineage blood progenitors by inducing ectopic OCT4 expression of the fibroblasts-derived CD45+ cells, promoting the transdifferentiation results from unipotent to multipotent [77]. After then, new strategies in the generation of hematopoietic progenitors 
emerged and were gradually optimized by either combining various transcription factors [78, 79] or facilitated with miRNA [80]. Szabo et al. also showed the in vivo engraftment capacity of these fibroblasts-derived blood progenitors comparable to that of umbilic blood and mobilized peripheral bloodderived progenitors. More efforts were made to derive engraftable and immunologically functional cells, such as conversion into immunocompetent HSCs [81] and antigen-presenting dendritic cells [82].

The procedure of direct reprogramming fully differentiated cells to either multilineage or single lineage hematopoietic progenitors in vitro are solid, but the in vivo engraftment capability can be further improved and developed for clinical use. One of the major hurdles is the difficulty of delivering reagents that mediate the conversions between distinct cell types. In a 2019 study, Chang et al. presented an efficient and safe in vivo direct conversion of fibroblasts into cardiomyocytes. The critical factors of cardiac reprogramming, GMT, were loaded in cationic gold nanoparticles delivered locally into mouse hearts [83]. The nanocarriers are promising candidates for a novel treatment for all kinds of diseases.

\section{TECHNOLOGIES ON TRANSDIFFERENTIATING}

Genome editing tools, including but not limited to bacterial artificial chromosome (BAC) based homologous recombination, zinc-finger nucleases (ZFNs), transcription activator-like effector nucleases (TALENs), and clustered regularly interspaced short palindromic repeat (CRISPR)-Cas9 nuclease systems, have been applied in stem cell and cellular differentiation studies [84]. In a 2010 study, ATM gene and p53 gene in human ESCs were targeted and disrupted by BAC-based vectors with large homologous arms, and by further differentiating these modified hESCs into fibroblasts, the responses of cells to DNA damage are characterized [85]. ZFNs consist of separate DNA-binding and FokI DNA-cleavage domains, and the binding recognition domain can be substituted to target different sequences and determine where to be cleaved by FokI [86]. Ramalingam et al. designed a ZFNmediated insertion of stem-cell transcription factor genes flanked by LoxP sites into the endogenous CCR5 locus of human lung fibroblasts and primary cord blood mononuclear cells, which converted them into iPSCs [87]. In particular, CRISPR/Cas system has shown its capability in inducing transdifferentiation of various cell types via either activation or inactivation of key regulators. Different from ZFNs and TALENs, the CRISPR/Cas system can cleave any DNA sequence by designing different crRNA, as long as there is a 'seed' sequence within the crRNA to be targeted by the Cas9 endonuclease and a protospacer adjacent motif sequence upstream of the crRNA-binding region [88]. The major advantage of using the CRISPR/Cas9 system for direct cell reprogramming is it precisely modulates endogenous gene expression without leaving permanent genome mutation. In 2016, two studies reported conversion from fibroblasts into neuronal cells via CRISPR/Cas9 based methods, while Rubio et al. chose to inactivate TSC2 gene [18] and Black et al. chose to activate the endogenous BAM factors by CRIPSR activation(CRISPR a) [89]. CRISPRa is a process in which the catalytically dead variant of Cas9, called dCas9, was directed to the promoter regions by fusing with transcriptional activators, affecting gene expression. In 2014, Chakraborty et al. first designed an enhanced robust CRISPRa system by fusing two transactivation domains to Cas9. This RNAguided ${ }^{\mathrm{VP} 64} \mathrm{dCas} 9-\mathrm{BFP}{ }^{\mathrm{VP} 64}$ fusion protein activated endogenous Myod1 in mouse embryonic fibroblasts and converted them into skeletal myocytes with a myogenic gene expression comparable to other Myod1-based transdifferentiation mentioned earlier [90]. Besides conversion from human fibroblasts into neuronal cells, CRISPR activators were also reported to perform reprogramming from fibroblasts into iPSCs and cardiac progenitor cells in recent studies [91, 92]. Moreover, CRISPRa system can also help locate key gene factors in cell fate changing by genome-scale screening. For example, in 2018, Liu et al. developed an approach that generates individual factor map and factor genetic interaction map after genome screening via CRISPRa. By further validations with top key genes on the maps, they discovered a novel combination of Ezh2 and Mecom, promoting transdifferentiation from fibroblasts into neuronal cells [93]. On the other hand, Lee and Chung have suggested to use gene-editing tools to perform correction on mutated genes and induction of transdifferentiation simultaneously, which ideally results in massive gene-corrected cells for tissue regeneration therapy [84].

Understanding the mechanism behind cell lineages changes also helps researchers revise their current protocols and discover new pathways of 
direct cell reprogramming. This can be assisted with the detailed characterization of cells using nextgeneration sequencing technologies. In 2009, Tang et al. were the first to report a high-throughput mRNA-seq assay for whole-transcriptome analysis on the single cell level [94]. Compared to bulk RNA-sequencing, which usually requires hundreds or thousands of cells and measures the average expression level of their genes, single-cell RNA-sequencing(scRNA-seq) provides an unbiased measurement of the heterogeneity within a cell population. ScRNA-seq consists of three main steps, isolation of individual cells, conversion of cellular RNA into cDNA, and parallel sequencing of cDNA libraries [95]. Benefited from the rapid development of modern scientific instruments, these three main technologies' protocols were optimized, resulting in faster and cheaper scRNA-seq assays. scRNA-seq were therefore broadly implemented in various field to facilitate researchers' studies, including stem cells studies. Revealing the trajectories behind cell type changes provides knowledge on both normal tissue formation and disease pathologies [96], which is deepened by performing scRNA-seq analysis at different time points throughout cell development. The comprehensive mapping of the trajectories also gives insights on novel transdifferentiation pathways. In a 2014 study, Quake's group performed scRNAseq on the mouse lung epithelial cells and identified alveolar, bronchiolar, and progenitor cell populations based on the hierarchical clustering of RNA-seq data [97]. Their data revealed discovery of novel markers in different cell populations, which can be used to distinguish cell types and suggest new strategies for conversion between different cell types, for example, from flat alveolar type 1 cells to cuboidal alveolar type 2 cells. Two years later, led again by Quake, the group dissected the transdifferentiation process from fibroblast to neuron using scRNA-seq [98]. Based on the sequencing data obtained at each time point, they were able to draw conclusions on the effects of Ascl1 overexpression in the initial condition and describe specific pathways and various stages these cells experienced during the transdifferentiation. Similarly, a study in 2018 analyzed the conversion from human brain pericytes into neurons by forcing the overexpression of Ascl1 and Sox2 using scRNA-seq [99]. They reconstructed the trajectories behind the process and uncovered the intermediate states by analyzing the scRNA-seq data using Monocle 2 package. Developed by Qiu et al. in 2017, Monocle 2 is an algorithm that better identifies branch points of cell fate decisions than its previous version [100]. Revealing the conversion barriers that cells were facing during the classic MyoD-mediated myogenic conversion, the algorithm has shown its capability in "debugging" the conversion process and discovering novel key determinants [101]. One major obstacle behind the trajectory reconstruction was the loss of lineage relationships during the process. Interestingly, Biddy et al. came up with a cell-indexing methodology called 'CellTagging', enabling single-cell profiling of cell identity and clonal history [102]. They used the system to reveal trajectories behind conversion from fibroblast to induced endoderm progenitors and found an essential component, Mettl7al, associated with the successful conversions. More recently, by comparing the trajectories of transdifferentiation and reprogramming from pre-B cells at the single-cell level, Francesconi et al. identified distinct cell subsets in the starting cell population, among which the small pre-BII cells transdifferentiate more rapidly than the large pre-BII cells. By distinguishing and targeting the cell subset that responds better during transdifferentiation induction, we will potentially improve the efficiency of transdifferentiation process and further promote its practicability as clinical trials [103].

\section{CONCLUSION AND FUTURE PERSPECTIVES}

Transdifferentiation into neuronal cells, skeletal myocytes, cardiac myocytes, and hematopoietic cells have been explored massively. Mature protocols existed for in vitro conversion from mouse and human cell sources; however, more investigation is needed for in vivo cases. Researchers have put extensive efforts into improving conversion efficiency by either overexpressing different transcription factors, transducing miRNAs into the system, or incorporating small molecules. Facilitating the next-generation biotechnologies and illustrating the signaling pathway behind each cell fate decision helps us recognize the critical factors and manipulate the transdifferentiation results.

However, knowledge is lacking to induce transdifferentiation in cell lineages with fewer findings, such as lung cells, hepatocytes, pancreatic cells, and other cell types derived from endoderm. This could be due to the inefficient way of finding the critical factors and the optimal combinations. Recently, an emerging technology, the CRISPR system, could improve the process by systematically screening to 
find the key factors. It has already been used in systematically identifying factors that promotes direct reprogramming from fibroblasts into neuronal cells [93]. Similarly, it is promising to apply this technology in various cell types. The next-generation sequencing analysis provides a comprehensive mapping of cell lineage trajectories, revealing hidden stages between cell type conversion. The potential discoveries may explain the formation of diseases and provide insight into new therapeutic strategies. At the same time, genome editing tools have revealed their capability in targeting essential genes and enhancing the efficiency of transdifferentiating into mature and functional cells. By further investigations and collaborations with other fields, transdifferentiation can be applied for clinical use in the foreseeable future.

\section{ACKNOWLEDGMENTS}

This work was supported by NCI R00 CA22635301A1, Cancer Research Foundation-Young Investigator Award, Jane D. Rowley Discovery Fund, and CTSA-ITM Core Subsidies Funding to HJC.

\section{CONFLICT OF INTEREST/DISCLOSURE STATEMENT}

The authors have no conflict of interest to report.

\section{STEMJOURNAL OPEN REVIEW}

The evaluations from peer reviewers for this article are freely available and can be found as supplementary material here: http://dx.doi.org/10.3233/ STJ-200003.

\section{REFERENCES}

[1] Ilic D, Ogilvie C. Concise Review: Human Embryonic Stem Cells-What Have We Done? What Are We Doing? Where Are We Going? STEM CELLS. 2017;35: 17-25.

[2] Takahashi K, Yamanaka S. Induction of Pluripotent Stem Cells from Mouse Embryonic and Adult Fibroblast Cultures by Defined Factors. Cell. 2006;126:663-76.

[3] Liao J, Wu Z, Wang Y, et al. Enhanced efficiency of generating induced pluripotent stem (iPS) cells from human somatic cells by a combination of six transcription factors. Cell Res. 2008;18:600-3.
[4] Nakagawa M, Koyanagi M, Tanabe K, et al. Generation of induced pluripotent stem cells without Myc from mouse and human fibroblasts. Nat Biotechnol. 2008;26:101-6.

[5] Ward C, Volpe G, Cauchy P, et al. Fine-Tuning Mybl2 Is Required for Proper Mesenchymal-to-Epithelial Transition during Somatic Reprogramming. Cell Rep. 2018;24:1496-1511.e8.

[6] Takahashi K, Tanabe K, Ohnuki M, et al. Induction of pluripotent stem cells from adult human fibroblasts by defined factors. Cell. 2007;131:861-72.

[7] Yu J, Vodyanik MA, Smuga-Otto K, et al. Induced Pluripotent Stem Cell Lines Derived from Human Somatic Cells. Science. 2007;318:1917-20.

[8] Seki T, Yuasa S, Fukuda K. Generation of induced pluripotent stem cells from a small amount of human peripheral blood using a combination of activated T cells and Sendai virus. Nat Protoc. 2012;7:718-28.

[9] Aasen T, Raya A, Barrero MJ, et al. Efficient and rapid generation of induced pluripotent stem cells from human keratinocytes. Nat Biotechnol. 2008;26: 1276-84.

[10] Xue Y, Cai X, Wang L, et al. Generating a Non-Integrating Human Induced Pluripotent Stem Cell Bank from UrineDerived Cells. PLOS ONE. 2013;8:e70573.

[11] Zhang G, Shang B, Yang P, et al. Induced pluripotent stem cell consensus genes: implication for the risk of tumorigenesis and cancers in induced pluripotent stem cell therapy. Stem Cells Dev. 2012;21:955-64.

[12] Jin $Y$, Shen $Y$, Weintraub NL, et al. Using iRFP Genetic Labeling Technology to Track Tumorogenesis of Transplanted CRISPR/Cas9-Edited iPSC in Skeletal Muscle. In: Basel MT, Bossmann SH (eds) Cell Tracking: Methods and Protocols. New York, NY: Springer US, pp. 73-83.

[13] Okada M, Tada Y, Seki T, et al. P1849Prevention of tumorigenesis in human pluripotent stem cell-derived cardiomyocytes by immunological cytotoxicity against oncofetal antigen. Eur Heart J; 39. Epub ahead of print 1 August 2018. DOI: 10.1093/eurheartj/ehy565.P1849.

[14] Davis RL, Weintraub H, Lassar AB. Expression of a single transfected cDNA converts fibroblasts to myoblasts. Cell. 1987;51:987-1000.

[15] Grath A, Dai G. Direct cell reprogramming for tissue engineering and regenerative medicine. J Biol Eng. Epub ahead of print. 2019. DOI: 10.1186/s13036-019-0144-9.

[16] Ahrens TD, Caglayan S, Staerk J, et al. Chapter 4 Transdifferentiation-Changing Cell Identity. In: Stem Cells and Biomaterials for Regenerative Medicine, pp. 37-56.

[17] Pournasr B, Khaloughi K, Salekdeh GH, et al. Concise review: alchemy of biology: generating desired cell types from abundant and accessible cells. Stem Cells Dayt Ohio. 2011;29:1933-41.

[18] Rubio A, Luoni M, Giannelli SG, et al. Rapid and efficient CRISPR/Cas9 gene inactivation in human neurons during human pluripotent stem cell differentiation and direct reprogramming. Sci Rep. 2016;6:37540.

[19] Jiang H, Xu Z, Zhong P, et al. Cell cycle and p53 gate the direct conversion of human fibroblasts to dopaminergic neurons. Nat Commun. 2015;6:10100.

[20] Wiegand C, Banerjee I. Recent advances in the applications of iPSC technology. Curr Opin Biotechnol. 2019;60:250-8. 
[21] Feng J. Kinetic barriers in transdifferentiation. Cell Cycle. 2016;15:1019-20.

[22] Vierbuchen T, Ostermeier A, Pang ZP, et al. Direct conversion of fibroblasts to functional neurons by defined factors. Nature. 2010;463:1035-41.

[23] Ambasudhan R, Talantova M, Coleman R, et al. Direct reprogramming of adult human fibroblasts to functional neurons under defined conditions. Cell Stem Cell. 2011;9:113-8.

[24] Pang ZP, Yang N, Vierbuchen T, et al. Induction of human neuronal cells by defined transcription factors. Nature. 2011;476:220-3

[25] Yoo AS, Sun AX, Li L, et al. MicroRNA-mediated conversion of human fibroblasts to neurons. Nature. 2011;476:228-31.

[26] Ladewig J, Mertens J, Kesavan J, et al. Small molecules enable highly efficient neuronal conversion of human fibroblasts. Nat Methods. 2012;9:575-8.

[27] Pfisterer U, Kirkeby A, Torper O, et al. Direct conversion of human fibroblasts to dopaminergic neurons. Proc Natl Acad Sci. 2011;108:10343-8.

[28] Son EY, Ichida JK, Wainger BJ, et al. Conversion of mouse and human fibroblasts into functional spinal motor neurons. Cell Stem Cell. 2011;9:205-18.

[29] Colasante G, Lignani G, Rubio A, et al. Rapid Conversion of Fibroblasts into Functional Forebrain GABAergic Interneurons by Direct Genetic Reprogramming. Cell Stem Cell. 2015;17:719-34.

[30] Mollinari C, Zhao J, Lupacchini L, et al. Transdifferentiation: a new promise for neurodegenerative diseases. Cell Death Dis. 2018;9:1-9.

[31] Tanabe K, Ang CE, Chanda S, et al. Transdifferentiation of human adult peripheral blood T cells into neurons. Proc Natl Acad Sci. 2018;115:6470-5.

[32] Han DW, Tapia N, Hermann A, et al. Direct reprogramming of fibroblasts into neural stem cells by defined factors. Cell Stem Cell. 2012;10:465-72.

[33] Thier M, Wörsdörfer P, Lakes YB, et al. Direct conversion of fibroblasts into stably expandable neural stem cells. Cell Stem Cell. 2012;10:473-9.

[34] Lujan E, Chanda S, Ahlenius H, et al. Direct conversion of mouse fibroblasts to self-renewing, tripotent neural precursor cells. Proc Natl Acad Sci. 2012;109: 2527-32.

[35] Ring KL, Tong LM, Balestra ME, et al. Direct reprogramming of mouse and human fibroblasts into multipotent neural stem cells with a single factor. Cell Stem Cell. 2012;11:100-9.

[36] Zhang M, Lin Y-H, Sun YJ, et al. Pharmacological Reprogramming of Fibroblasts into Neural Stem Cells by Signaling-Directed Transcriptional Activation. Cell Stem Cell. 2016;18:653-67.

[37] Capetian P, Azmitia L, Pauly MG, et al. PlasmidBased Generation of Induced Neural Stem Cells from Adult Human Fibroblasts. Front Cell Neurosci; 10. Epub ahead of print 24 October. 2016. DOI: 10.3389/fncel.2016.00245.

[38] Huang P, He Z, Ji S, et al. Induction of functional hepatocyte-like cells from mouse fibroblasts by defined factors. Nature. 2011;475:386-9.

[39] Sekiya S, Suzuki A. Direct conversion of mouse fibroblasts to hepatocyte-like cells by defined factors. Nature. 2011;475:390-3.
[40] Yu B, He Z-Y, You P, et al. Reprogramming fibroblasts into bipotential hepatic stem cells by defined factors. Cell Stem Cell. 2013;13:328-40.

[41] Huang P, Zhang L, Gao Y, et al. Direct Reprogramming of Human Fibroblasts to Functional and Expandable Hepatocytes. Cell Stem Cell. 2014;14:370-84.

[42] Kim J, Kim K-P, Lim KT, et al. Generation of integrationfree induced hepatocyte-like cells from mouse fibroblasts. Sci Rep. 2015;5:15706.

[43] Simeonov KP, Uppal H. Direct reprogramming of human fibroblasts to hepatocyte-like cells by synthetic modified mRNAs. PloS One. 2014;9:e100134.

[44] Nakamori D, Akamine H, Takayama K, et al. Direct conversion of human fibroblasts into hepatocyte-like cells by ATF5, PROX1, FOXA2, FOXA3, and HNF4A transduction. Sci Rep. 2017;7:16675.

[45] Song G, Pacher M, Balakrishnan A, et al. Direct Reprogramming of Hepatic Myofibroblasts into Hepatocytes In Vivo Attenuates Liver Fibrosis. Cell Stem Cell. 2016;18:797-808.

[46] Puri S, Folias AE, Hebrok M. Plasticity and dedifferentiation within the pancreas: development, homeostasis, and disease. Cell Stem Cell. 2015;16:18-31.

[47] Zhou Q, Brown J, Kanarek A, et al. In vivo reprogramming of adult pancreatic exocrine cells to $\beta$-cells. Nature. 2008;455:627-32.

[48] Li W, Nakanishi M, Zumsteg A, et al. In vivo reprogramming of pancreatic acinar cells to three islet endocrine subtypes. eLife. 2014;3:e1846.

[49] Graf T. Historical Origins of Transdifferentiation and Reprogramming. Cell Stem Cell. 2011;9:504-16.

[50] Taylor SM, Jones PA. Multiple new phenotypes induced in 10T1/2 and 3T3 cells treated with 5-azacytidine. Cell. 1979;17:771-9.

[51] Tapscott SJ, Davis RL, Thayer MJ, et al. MyoD1: a nuclear phosphoprotein requiring a Myc homology region to convert fibroblasts to myoblasts. Science. 1988;242: 405-11.

[52] Weintraub H, Tapscott SJ, Davis RL, et al. Activation of muscle-specific genes in pigment, nerve, fat, liver, and fibroblast cell lines by forced expression of MyoD. Proc Natl Acad Sci U S A. 1989;86:5434-8.

[53] Boukamp P, Chen J, Gonzales F, et al. Progressive stages of 'transdifferentiation' from epidermal to mesenchymal phenotype induced by MyoD1 transfection, 5-aza-2'deoxycytidine treatment, and selection for reduced cell attachment in the human keratinocyte line HaCaT. J Cell Biol. 1992;116:1257-71.

[54] Lattanzi L, Salvatori G, Coletta M, et al. High efficiency myogenic conversion of human fibroblasts by adenoviral vector-mediated MyoD gene transfer. An alternative strategy for ex vivo gene therapy of primary myopathies. J Clin Invest. 1998;101:2119-28.

[55] Liu Z, Fan H, Li Y, et al. Experimental Studies on the Differentiation of Fibroblasts into Myoblasts induced by MyoD Genes in vitro. Int J Biomed Sci IJBS. 2008;4:14-9.

[56] Del Bo R, Torrente $\mathrm{Y}$, Corti S, et al. In vitro and in vivo tetracycline-controlled myogenic conversion of NIH-3T3 cells: evidence of programmed cell death after muscle cell transplantation. Cell Transplant. 2001;10:209-21.

[57] Bichsel C, Neeld D, Hamazaki T, et al. Direct Reprogramming of Fibroblasts to Myocytes via Bacterial Injection of MyoD Protein. Cell Reprogramming. 2013;15:117-25. 
[58] Boularaoui SM, Abdel-Raouf KMA, Alwahab NSA, et al. Efficient transdifferentiation of human dermal fibroblasts into skeletal muscle. J Tissue Eng Regen Med. 2018;12:e918-e936.

[59] Ito N, Kii I, Shimizu N, et al. Direct reprogramming of fibroblasts into skeletal muscle progenitor cells by transcription factors enriched in undifferentiated subpopulation of satellite cells. Sci Rep. 2017;7:8097.

[60] Lee E-J, Kim M, Kim YD, et al. Establishment of stably expandable induced myogenic stem cells by four transcription factors. Cell Death Dis. 2018;9:1-18.

[61] Hwang M, Lee E-J, Chung M-J, et al. Five transcriptional factors reprogram fibroblast into myogenic lineage cells via paraxial mesoderm stage. Cell Cycle. 2020;19: 1804-16.

[62] Sadahiro T. Cardiac regeneration with pluripotent stem cell-derived cardiomyocytes and direct cardiac reprogramming. Regen Ther. 2019;11:95-100.

[63] Klose K, Gossen M, Stamm C. Turning fibroblasts into cardiomyocytes: technological review of cardiac transdifferentiation strategies. FASEB J Off Publ Fed Am Soc Exp Biol. 2019;33:49-70.

[64] Ieda M, Fu J-D, Delgado-Olguin P, et al. Direct reprogramming of fibroblasts into functional cardiomyocytes by defined factors. Cell. 2010;142:375-86.

[65] Tani H, Sadahiro T, Ieda M. Direct Cardiac Reprogramming: A Novel Approach for Heart Regeneration. Int $\mathbf{J}$ Mol Sci; 19. Epub ahead of print 5 September 2018. DOI: 10.3390/ijms19092629.

[66] Hirai H, Katoku-Kikyo N, Keirstead SA, et al. Accelerated direct reprogramming of fibroblasts into cardiomyocytelike cells with the MyoD transactivation domain. Cardiovasc Res. 2013;100:105-13.

[67] Jayawardena TM, Finch EA, Zhang L, et al. MicroRNA induced cardiac reprogramming in vivo: evidence for mature cardiac myocytes and improved cardiac function. Circ Res. 2015;116:418-24.

[68] Fu Y, Huang C, Xu X, et al. Direct reprogramming of mouse fibroblasts into cardiomyocytes with chemical cocktails. Cell Res. 2015;25:1013-24.

[69] Boyd AW, Schrader JW. Derivation of macrophage-like lines from the pre-B lymphoma ABLS 8.1 using 5azacytidine. Nature. 1982;297:691-3.

[70] Graf T. Differentiation plasticity of hematopoietic cells. Blood. 2002;99:3089-101.

[71] Xie H, Ye M, Feng R, et al. Stepwise Reprogramming of B Cells into Macrophages. Cell. 2004;117:663-76.

[72] Nutt SL, Heavey B, Rolink AG, et al. Commitment to the B-lymphoid lineage depends on the transcription factor Pax5. Nature. 1999;401:556-62.

[73] Rolink AG, Nutt SL, Melchers F, et al. Long-term in vivo reconstitution of T-cell development by Pax5-deficient Bcell progenitors. Nature. 1999;401:603-6.

[74] DeKoter RP, Singh H. Regulation of B lymphocyte and macrophage development by graded expression of PU.1. Science. 2000;288:1439-41.

[75] Laiosa CV, Stadtfeld M, Xie H, et al. Reprogramming of Committed T Cell Progenitors to Macrophages and Dendritic Cells by C/EBP $\alpha$ and PU.1 Transcription Factors. Immunity. 2006;25:731-44.

[76] Feng R, Desbordes SC, Xie H, et al. PU.1 and C/EBP $\alpha / \beta$ convert fibroblasts into macrophage-like cells. Proc Natl Acad Sci. 2008;105:6057-62.
[77] Szabo E, Rampalli S, Risueño RM, et al. Direct conversion of human fibroblasts to multilineage blood progenitors. Nature. 2010;468:521-6.

[78] Durán JGB, Lis R, Rafii S. Haematopoietic stem cell reprogramming and the hope for a universal blood product. FEBS Lett. Epub ahead of print. 2019. DOI: 10.1002/1873-3468.13681.

[79] Batta K, Florkowska M, Kouskoff V, et al. Direct reprogramming of murine fibroblasts to hematopoietic progenitor cells. Cell Rep. 2014;9:1871-84.

[80] Pulecio J, Nivet E, Sancho-Martinez I, et al. Conversion of human fibroblasts into monocyte-like progenitor cells. Stem Cells Dayt Ohio. 2014;32:2923-38.

[81] Lis R, Karrasch CC, Poulos MG, et al. Conversion of adult endothelium to immunocompetent haematopoietic stem cells. Nature. 2017;545:439-45.

[82] Rosa FF, Pires CF, Kurochkin I, et al. Direct reprogramming of fibroblasts into antigen-presenting dendritic cells. Sci Immunol; 3. Epub ahead of print 7 December. 2018. DOI: 10.1126/sciimmunol.aau4292.

[83] Chang Y, Lee E, Kim J, et al. Efficient in vivo direct conversion of fibroblasts into cardiomyocytes using a nanoparticle-based gene carrier. Biomaterials. 2019;192:500-9.

[84] Lee S-Y, Chung S-K. Integrating Gene Correction in the Reprogramming and Transdifferentiation Processes: A One-Step Strategy to Overcome Stem Cell-Based Gene Therapy Limitations. Stem Cells Int; 2016. Epub ahead of print. 2016. DOI: $10.1155 / 2016 / 2725670$.

[85] Song H, Chung S-K, Xu Y. Modeling disease in human ESCs using an efficient BAC-based homologous recombination system. Cell Stem Cell. 2010;6:80-9.

[86] Gaj T, Gersbach CA, Barbas CF. ZFN, TALEN, and CRISPR/Cas-based methods for genome engineering. Trends Biotechnol. 2013;31:397-405.

[87] Ramalingam S, London V, Kandavelou K, et al. Generation and genetic engineering of human induced pluripotent stem cells using designed zinc finger nucleases. Stem Cells Dev. 2013;22:595-610.

[88] Jinek M, Chylinski K, Fonfara I, et al. A Programmable Dual-RNA-Guided DNA Endonuclease in Adaptive Bacterial Immunity. Science. 2012;337:816-21.

[89] Black JB, Adler AF, Wang H-G, et al. Targeted Epigenetic Remodeling of Endogenous Loci by CRISPR/Cas9-Based Transcriptional Activators Directly Converts Fibroblasts to Neuronal Cells. Cell Stem Cell. 2016;19:406-14.

[90] Chakraborty S, Ji H, Kabadi AM, et al. A CRISPR/Cas9Based System for Reprogramming Cell Lineage Specification. Stem Cell Rep. 2014;3:940-7.

[91] Wang J, Jiang X, Zhao L, et al. Lineage reprogramming of fibroblasts into induced cardiac progenitor cells by CRISPR/Cas9-based transcriptional activators. Acta Pharm Sin B. 2020;10:313-26.

[92] Weltner J, Balboa D, Katayama S, et al. Human pluripotent reprogramming with CRISPR activators. Nat Commun. 2018;9:2643

[93] Liu Y, Yu C, Daley TP, et al. CRISPR Activation Screens Systematically Identify Factors that Drive Neuronal Fate and Reprogramming. Cell Stem Cell. 2018;23:758771.e8.

[94] Tang F, Barbacioru C, Wang Y, et al. mRNA-Seq wholetranscriptome analysis of a single cell. Nat Methods. 2009;6:377-82. 
[95] Saliba A-E, Westermann AJ, Gorski SA, et al. Single-cell RNA-seq: advances and future challenges. Nucleic Acids Res. 2014;42:8845-60.

[96] Kester L, van Oudenaarden A. Single-Cell Transcriptomics Meets Lineage Tracing. Cell Stem Cell. 2018;23:166-79.

[97] Treutlein B, Brownfield DG, Wu AR, et al. Reconstructing lineage hierarchies of the distal lung epithelium using single cell RNA-seq. Nature. 2014;509:371-5.

[98] Treutlein B, Lee QY, Camp JG, et al. Dissecting direct reprogramming from fibroblast to neuron using single-cell RNA-seq. Nature. 2016;534:391-5.

[99] Karow M, Camp JG, Falk S, et al. Direct pericyte-toneuron reprogramming via unfolding of a neural stem cell-like program. Nat Neurosci. 2018;21:932-40.
[100] Qiu X, Mao Q, Tang Y, et al. Reversed graph embedding resolves complex single-cell trajectories. Nat Methods. 2017;14:979-82.

[101] Cacchiarelli D, Qiu X, Srivatsan S, et al. Aligning SingleCell Developmental and Reprogramming Trajectories Identifies Molecular Determinants of Myogenic Reprogramming Outcome. Cell Syst. 2018;7:258-268.e3.

[102] Biddy BA, Kong W, Kamimoto K, et al. Single-cell mapping of lineage and identity in direct reprogramming. Nature. 2018;564:219-24.

[103] Francesconi M, Di Stefano B, Berenguer C, et al. Single cell RNA-seq identifies the origins of heterogeneity in efficient cell transdifferentiation and reprogramming. eLife. 2019;8:e41627. 